\title{
Single-spin asymmetry in pion production in polarized proton-proton collisions and odderon
}

\author{
A. Ahmedov ${ }^{1}$, E. N. Antonov ${ }^{2}$, E. Bartoš ${ }^{1 *}$, \\ E. A. Kuraev ${ }^{1}$ and E. Zemlyanaya ${ }^{1}$ \\ 1 Join Institute for Nuclear Research, 141980 Dubna, Russia, \\ ${ }^{2}$ Leningrad Institute for Nuclear Physics, Gatchina, Russia
}

\begin{abstract}
Single-spin asymmetry appears due to the interference of single and double gluon exchange between protons. A heavy fermion model is used to describe the jet production in the interaction of gluon with the proton implying the further averaging over its mass. As usually in one-spin correlations, the imaginary part of the double gluon exchange amplitude play the relevant role. The asymmetry in the inclusive setup with the pion tagged in the fragmentation region of the polarized proton does not depend on the center of mass energy in the limits of its large values. The lowest order radiative corrections to the polarized and unpolarized contributions to the differential cross sections are calculated in the leading logarithmical approximation. In general, a coefficient at logarithm of the ratio of cms energy to the pion mass depends on transversal momentum of the pion. This ratio of the lowest order contribution to the asymmetry may be interpreted as the partial contribution to the odderon intercept. The ratio of the relevant contributions in the unpolarized case can be associated with the partial contribution to the pomeron intercept. The numerical results given for the model describe the jet as a heavy fermion decay fragments.
\end{abstract}

${ }^{*}$ On leave of absence from Comenius University, 84248 Bratislava, Slovakia 


\section{Introduction}

Let us consider the inclusive process of the pion creation in the fragmentation region of polarized proton at high energy proton-proton collisions

$$
P_{1}\left(p_{1}, a\right)+P_{2}\left(p_{2}\right) \rightarrow \pi(p)+X_{1}\left(p_{1}^{\prime}\right)+X_{2}\left(p_{2}^{\prime}\right),
$$

where $a$ is the transversal to beam (implied by cms) axes spin of initial proton

$$
\begin{gathered}
a=(0,0, \mathbf{a}), \quad p=(E \beta, E \beta, \mathbf{p}), \quad E=\sqrt{s} / 2, \quad s=\left(p_{1}+p_{2}\right)^{2} \gg \mathbf{p}^{2} \sim m^{2}, \\
p_{1}=E\left(1, \beta_{0}, 0,0\right), \quad p_{2}=E\left(1,-\beta_{0}, 0,0\right), \quad \beta_{0}=\sqrt{1-\frac{m^{2}}{E^{2}}}, \quad
\end{gathered}
$$

where $\beta \sim 1$ is the energy fraction of pion, $M_{1,2}=\sqrt{p_{1,2}^{\prime 2}}$ are the invariant masses of the jets, which we will assume to be of the order of nucleon mass $m$. We study the two jet kinematics with jets $X_{1}, X_{2}$ moving along the initial hadron directions. The jet created by the transversely polarized proton is supposed to contain the detected pion. Moreover, we consider the case when its production is not related with the creation of nucleon resonances. In terms of pion transverse components it corresponds to the condition

$$
\begin{gathered}
\tilde{s}_{1}=\left(p+p_{1}^{\prime}\right)^{2}=\frac{1}{\beta \bar{\beta}}\left[\beta M_{1}^{2}+\left(\mathbf{p}+\beta \mathbf{k}_{\mathbf{1}}\right)^{2}\right]>M_{\text {res }}^{2}-m^{2}, \\
\mathbf{p}+\mathbf{k}_{\mathbf{1}}+\mathbf{p}_{\mathbf{1}}^{\prime}=0,
\end{gathered}
$$

where $\mathbf{k}_{\mathbf{1}}$ is the transfer momentum between protons. Through this paper we use Sudakov parameterization of 4 -momenta of the problem

$$
k_{i}=\alpha_{i} q_{2}+\beta_{i} q_{1}+k_{i \perp}, \quad k_{i \perp}=\left(0,0, \mathbf{k}_{\mathbf{i}}\right), \quad q_{1,2}=E(1, \pm 1,0,0) .
$$

The azimuthal one-spin asymmetry arises from the interference of the amplitudes with one and two gluon exchanges between nucleons (see Fig. 1)

$$
\begin{gathered}
A=\frac{2 E_{\pi} \frac{d^{3} \sigma(\mathbf{a}, \mathbf{p})}{d^{3} p}-2 E_{\pi} \frac{d^{3} \sigma(-\mathbf{a}, \mathbf{p})}{d^{3} p}}{2 E_{\pi} \frac{d^{3} \sigma(\mathbf{a}, \mathbf{p})}{d^{3} p}+2 E_{\pi} \frac{d^{3} \sigma(-\mathbf{a}, \mathbf{p})}{d^{3} p}}=\alpha_{s} R f(\rho, z), \quad R=|\mathbf{a}| \sin \varphi \\
f(\rho, z)=\frac{I_{0}^{(3)} T_{0}+z I_{1}^{(3)} T_{1}}{J_{0}^{(2)} T_{2}+z J_{1}^{(2)} T_{3}}, \quad \rho=\frac{|\mathbf{p}|}{m}, \quad z=\frac{\alpha_{s}}{\pi} \ln \frac{s}{m^{2}}
\end{gathered}
$$

where $\alpha_{s}$ is the strong coupling constant, $\varphi$ is the azimuthal angle between the 2 -vectors $\mathbf{a}$ and $\mathbf{p}$, transverse to the beam axis. The functions $I, J$ as 

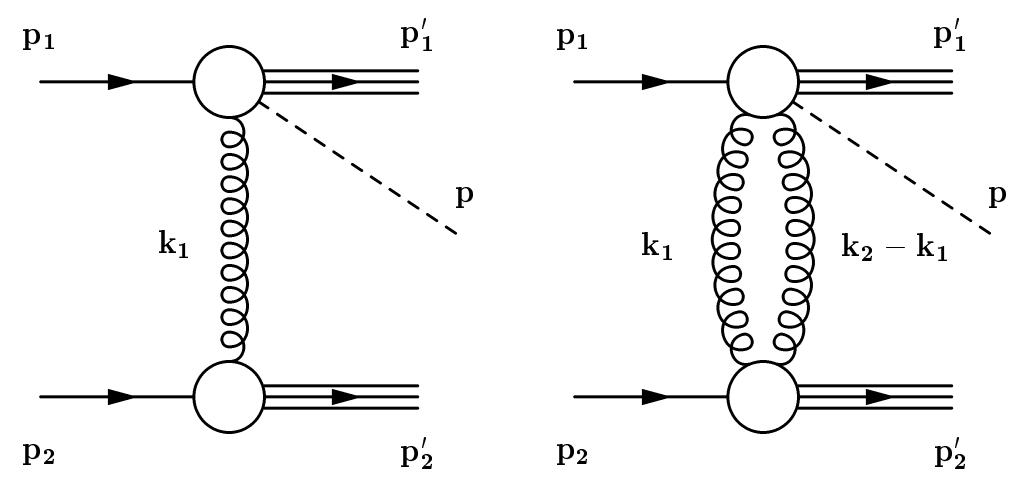

Fig. 1: One and two gluon exchange in peripheral collision of protons with $\pi$ production $P_{1}\left(p_{1}, a\right)+P_{2}\left(p_{2}\right) \rightarrow \pi(p)+X_{1}\left(p_{1}^{\prime}\right)+X_{2}\left(p_{2}^{\prime}\right)$.

well as the color factors $T_{i}$ will be specified below. For convenience we put here the alternative form for the phase volume of pion

$$
\frac{d^{3} p}{2 E_{\pi}}=m^{2} \rho d \rho d \phi \frac{d \beta}{2 \beta} .
$$

The one gluon exchange matrix element has a form

$$
M_{0}=i 4 \pi \alpha_{s} \frac{J_{\mu}^{(1 a)} J_{\nu}^{(2 a)} g_{\mu \nu}}{k_{1}^{2}}
$$

the currents $J^{(1 a)}, J^{(2 a)}$ are associated with the jets created by particles 1,2 and index $a$ describes the color state of the jet. Using Gribov representation of the metric tensor

$$
g_{\mu \nu} \approx \frac{2}{s} p_{2}^{\mu} p_{1}^{\nu},
$$

and the gauge condition for the currents

$$
k_{1}^{\mu} J_{\mu}^{(1 a)}=\left(\alpha_{1} p_{2}+k_{1 \perp}\right) J^{(1 a)}=0, \quad k_{1}^{\mu} J_{\mu}^{(2 a)}=\left(\beta_{1} p_{1}+k_{1 \perp}\right) J^{(2 a)}=0,
$$

we express $M_{0}$ in the form

$$
\begin{gathered}
M_{0}=i 8 s \pi \alpha_{s} \sum_{a=1}^{N^{2}-1} \frac{\mathbf{J}^{(\mathbf{1} \mathbf{a})} \cdot \mathbf{k}_{\mathbf{1}} \mathbf{J}^{(2 \mathbf{a})} \cdot \mathbf{k}_{\mathbf{1}}}{s_{1} s_{2} \mathbf{k}_{\mathbf{1}}{ }^{2}}, \\
s_{1}=-s \alpha_{1}=\tilde{s}_{1}+\mathbf{k}_{\mathbf{1}}{ }^{2}-m^{2}, \quad s_{2}=s \beta_{1}=M_{2}{ }^{2}-m^{2}+\mathbf{k}_{\mathbf{1}}{ }^{2} .
\end{gathered}
$$


The quantities $M_{1,2}$ are the invariant masses of the jets. We imply that the jet $X_{1}$ does not contain the detected pion. At this point we need some model describing the jets. We use the heavy fermion model, i.e., we consider the jet as a result of heavy fermion decay. We assume the coupling constant of the interaction of the pion with a nucleon and with heavy fermion to be the same. We do not specify it as well as it is cancelled in the asymmetry (5). So we have

$$
\begin{gathered}
J_{\mu}^{(1 a)}=\bar{u}\left(p_{1}^{\prime}\right) t^{a} O_{\mu} u\left(p_{1}\right), \quad J_{\mu}^{(2 a)}=\bar{u}\left(p_{2}^{\prime}\right) t^{a} \gamma_{\mu} u\left(p_{2}\right), \\
O_{\mu}=\gamma_{5} \frac{\hat{p}_{1}-\hat{k}_{1}+M_{1}}{d_{1}} \gamma_{\mu}+\gamma_{\mu} \frac{\hat{p}_{1}^{\prime}+\hat{k}_{1}+m}{d_{2}} \gamma_{5},
\end{gathered}
$$

$t^{a}$ are the generators of color group $S U(N)$ and

$$
d_{1}=\frac{1}{\beta \bar{\beta}}\left[\beta^{2} M_{1}^{2}+\left(\mathbf{p}+\beta \mathbf{k}_{\mathbf{1}}\right)^{2}\right], \quad d_{2}=-\frac{m^{2}}{\beta}\left[\rho^{2}+\beta^{2}\right] .
$$

We use here the spin density matrices of the jets (i.e., heavy fermions)

$$
\sum_{\lambda} u^{\lambda}\left(p_{1}^{\prime}\right) \bar{u}^{\lambda}\left(p_{1}^{\prime}\right)=\hat{p}_{1}^{\prime}+M_{1}, \quad \sum_{\lambda} u^{\lambda}\left(p_{2}^{\prime}\right) \bar{u}^{\lambda}\left(p_{2}^{\prime}\right)=\hat{p}_{2}^{\prime}+M_{2}
$$

It is important to note that we impose the gauge invariant form of matrix element and after that we use the heavy fermion model. This operations do not commute as well as the heavy fermion currents do not satisfy the current conservation condition. It is a specific of the considered model.

For the lowest order differential cross section we obtain

$$
2 E_{\pi} \frac{d^{3} \sigma(p)}{d^{3} p}=\frac{2 \alpha_{s}^{2}}{\pi^{2} \bar{\beta}} T_{2} \int \frac{d^{2} \mathbf{k}_{\mathbf{1}}}{\pi} \frac{\Phi_{01}\left(\mathbf{k}_{\mathbf{1}}, \mathbf{p}\right) \Phi_{02}\left(\mathbf{k}_{\mathbf{1}}\right)}{\left(\mathbf{k}_{\mathbf{1}}^{2}\right)^{2}}=\frac{2 \alpha_{s}^{2}}{\pi^{2} \bar{\beta}} T_{2} J_{0}^{(2)}
$$

with the explicit expressions for the impact factors $\Phi_{01}, \Phi_{02}$ given in Appendix $\mathrm{A}$ and $T_{2}=\left(N^{2}-1\right) / 4$.

The lowest order spin-dependent contribution to the cross section arises from the interference of imaginary part of 1-loop radiative correction ( $\mathrm{RC}$ ) of Feynman diagram (FD) Fig 1b,c with the Born amplitude Fig 1a. We do not consider the RC from FD Fig. 1, believing that such kind FD contribute to the nucleon resonances formation. Besides it do not contribute in the leading logarithmical approximation (LLA)

$$
z \sim 1, \quad \frac{\alpha_{s}}{\pi} \ll 1
$$


We obtain in the lowest order

$$
2 E_{\pi} \frac{d \sigma}{d^{3} p}=\frac{2 \alpha_{s}^{3}}{\pi^{2} \bar{\beta}} R T_{0} I_{0}^{(3)}
$$

with

$$
I_{0}^{(3)}=-\int \frac{d^{2} \mathbf{k}_{\mathbf{1}}}{\pi} \frac{d^{2} \mathbf{k}}{\pi} \frac{\Phi_{11} \Phi_{22}}{\mathbf{k}_{\mathbf{1}}{ }^{2} \mathbf{k}^{2}\left(\mathbf{k}_{\mathbf{1}}-\mathbf{k}\right)^{2}}
$$

and the color factor

$$
T_{0}=\left|\operatorname{Tr}\left(t^{a} t^{b} t^{c}\right)\right|^{2}=\frac{1}{16}\left[f_{a b c}^{2}+d_{a b c}^{2}\right]=\frac{\left(N^{2}-1\right)\left(N^{2}-2\right)}{8 N} .
$$

The impact factors $\Phi_{11,22}$ are given in Appendix B.

Impact factors $\Phi_{11,22}$ contain the new mass parameters $\tilde{M}_{1,2}$ which are intermediate jet state masses.

\section{Ladder expansion}

We had shown that the lowest order unpolarized and polarized cross sections can be expressed in terms of impact factors of projectiles moving in opposite directions which where introduced first in the papers of H. Cheng and $\mathrm{T} . \mathrm{T} . \mathrm{Wu}$ [目]. The calculation of $\mathrm{RC}$ to them can be done following the method developed by J. Balitski and L. N. Lipatov [2]. It was shown by these authors that in the LLA, the cross section has as well the form of conversion of impact factors of colliding particles with some universal kernel. Physically it corresponds to the replacement of exchanged gluons by the reggeized gluons. The reggeization states are taking into account in two factors. First the Regge factor $\left(s / m^{2}\right)^{a(t)}$ must be introduced, where $a(t)$ is the Regge trajectory of gluon with the momentum squared $t$. The second factor takes into account the contribution of inelastic processes of emission of real gluons. These both contributions suffer from the infrared divergences, however the total sum is free of them. For the $\mathrm{RC}$ to the unpolarized cross section we have

$$
2 E_{\pi} \frac{d \sigma}{d^{3} p}=\frac{2 \alpha_{s}^{3}}{\pi^{3} \bar{\beta}} T_{3} \ln \frac{s}{m^{2}} J_{1}^{(2)}
$$

with color factor

$$
T_{3}=\operatorname{Tr}\left(t^{a} t^{b}\right) \operatorname{Tr}\left(t^{a^{\prime}} t^{b^{\prime}}\right)\left(-f_{a a^{\prime} d} f_{b d b^{\prime}}\right)=\frac{N}{4}\left(N^{2}-1\right)
$$




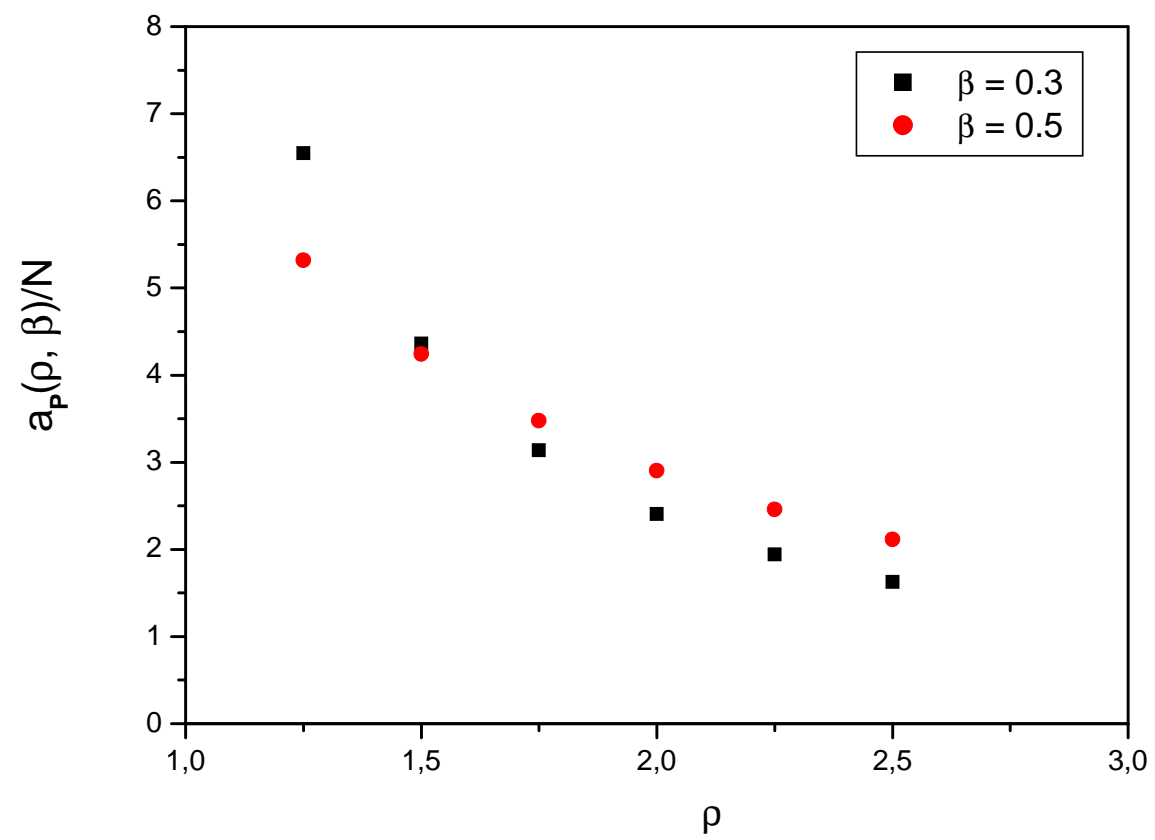

Fig. 2: The $\rho, \beta$ dependence of a partial contribution to the pomeron intercept.

and

$$
J_{1}^{(2)}=2 \int \frac{d^{2} \mathbf{k}_{\mathbf{1}}}{\pi} \frac{d^{2} \mathbf{k}^{\prime}}{\pi} \frac{\Phi_{1}\left(\mathbf{k}_{\mathbf{1}}, \mathbf{p}\right)}{\mathbf{k}_{\mathbf{1}}{ }^{2}\left(\mathbf{k}_{\mathbf{1}}-\mathbf{k}^{\prime}\right)^{2}}\left[\frac{\Phi_{2}\left(\mathbf{k}^{\prime}\right)}{\mathbf{k}^{\prime 2}}-\frac{\Phi_{2}\left(\mathbf{k}_{\mathbf{1}}\right)}{\mathbf{k}^{\prime 2}+\left(\mathbf{k}_{\mathbf{1}}-\mathbf{k}^{\prime}\right)^{2}}\right],
$$

with $\Phi_{1}$ and $\Phi_{2}$ given in Appendix C.

This formula can be inferred from the result for the non forward high energy scattering amplitude obtained in the paper [2]

$$
I_{1}=3 i s \frac{\alpha_{s}}{2} \ln \left(s / m^{2}\right) \int \frac{d^{2} \mathbf{k}}{\pi} \frac{\Phi^{A A^{\prime}}(\mathbf{k}, \mathbf{q})}{\mathbf{k}^{2}(\mathbf{q}-\mathbf{k})^{2}} \Phi^{B B^{\prime}} * K\left(\mathbf{k}, \mathbf{k}^{\prime}, \mathbf{q}\right),
$$

with definition

$$
\begin{aligned}
& \Phi^{B B^{\prime}} * K\left(\mathbf{k}, \mathbf{k}^{\prime}, \mathbf{q}\right)= \\
& \int \frac{d^{2} \mathbf{k}^{\prime}}{\pi}\left\{\left[-\mathbf{q}^{2}+\frac{\mathbf{k}^{2}\left(\mathbf{q}-\mathbf{k}^{\prime}\right)^{2}+\mathbf{k}^{\prime 2}(\mathbf{q}-\mathbf{k})^{2}}{\left(\mathbf{k}-\mathbf{k}^{\prime}\right)^{2}}\right] \frac{\Phi^{B B^{\prime}}\left(\mathbf{k}^{\prime}, \mathbf{q}\right)}{\mathbf{k}^{\prime 2}\left(\mathbf{q}-\mathbf{k}^{\prime}\right)^{2}}\right. \\
& \left.-\frac{\Phi^{B B^{\prime}}(\mathbf{k}, \mathbf{q})}{\left(\mathbf{k}-\mathbf{k}^{\prime}\right)^{2}}\left[\frac{\mathbf{q}-\mathbf{k})^{2}}{\mathbf{k}^{\prime 2}+\left(\mathbf{k}-\mathbf{k}^{\prime}\right)^{2}}+\frac{\left(\mathbf{q}-\mathbf{k}^{\prime}\right)^{2}+\left(\mathbf{k}-\mathbf{k}^{\prime}\right)^{2}}{(\mathbf{q}}\right]\right\} .
\end{aligned}
$$




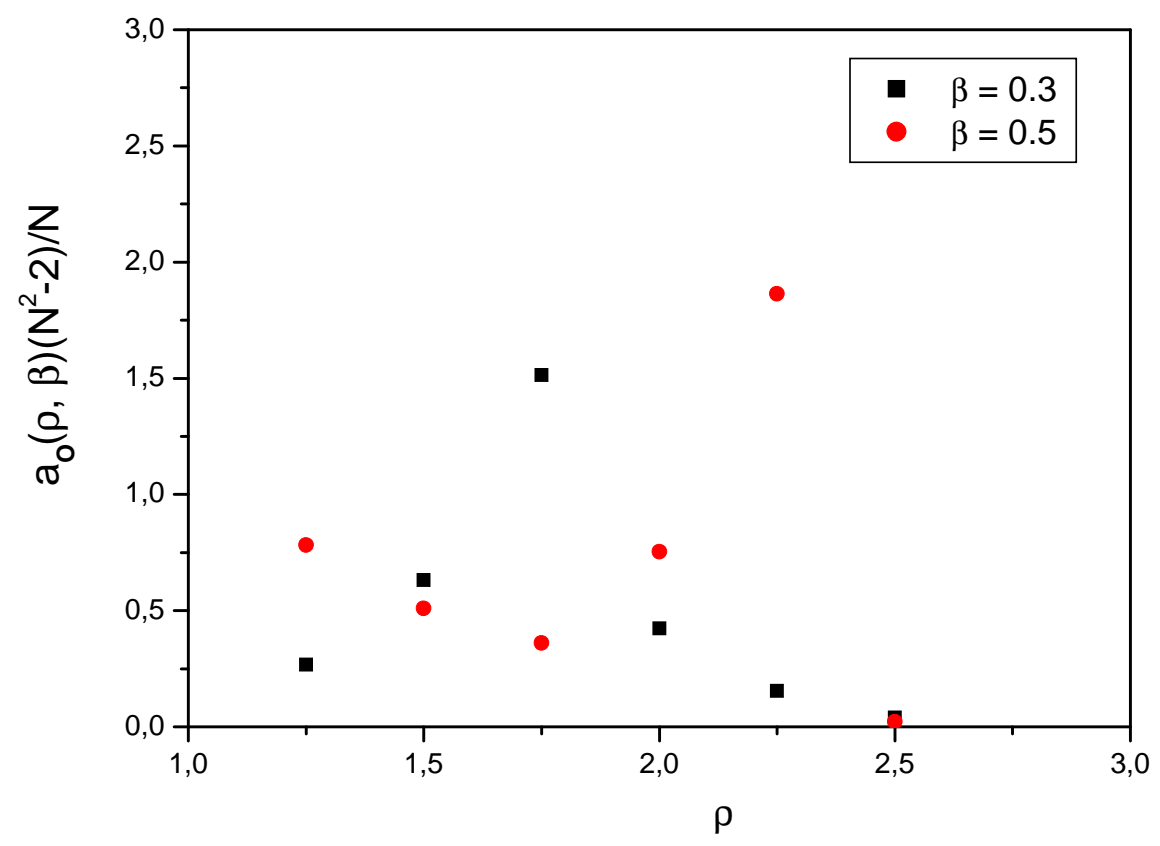

Fig. 3: The $\rho, \beta$ dependence of a partial contribution to the odderon intercept.

The formula (17) can be obtained from the last general one by putting $\mathbf{q}=0$. Note that in the formula obtained in [2] the used color group was $S U(2)$.

Let now consider the LLA RC to the polarized part of the differential cross section. There are presented three types of contributions corresponding to three different choices of two gluons which are involved in the reggeization procedure in the lowest order $\mathrm{RC}$

$$
2 E_{\pi} \frac{d \sigma}{d^{3} p}=\frac{\alpha_{s}^{4}}{\pi^{3}} R T_{1} I_{1}^{(3)} \ln \frac{s}{m^{2}}, \quad I_{1}^{(3)}=\int \frac{d^{2} \mathbf{k}}{\pi} \frac{d^{2} \mathbf{k}^{\prime}}{\pi} \frac{d^{2} \mathbf{k}_{1}}{\pi}\left[I_{12}+I_{13}+I_{23}\right],
$$

with the color factor

$$
T_{1}=\operatorname{Tr}^{a} t^{b} t^{c} \operatorname{Tr} t^{b^{\prime}} t^{a^{\prime}} t^{c} f_{a a^{\prime} d} f_{b d b^{\prime}}=\frac{\left(N^{2}-1\right)}{8} .
$$

Symmetry reasons lead to the conclusion that $I_{13}$ and $I_{23}$ contributions 
are equal to $I_{12}$ one. For $I_{12}$ we have

$$
\begin{aligned}
I_{12}= & \frac{\Phi_{1}^{(12)}\left(\mathbf{k}, \mathbf{k}_{\mathbf{1}}\right)}{\mathbf{k}^{2}\left(\mathbf{k}_{\mathbf{1}}-\mathbf{k}\right)^{2}}\left\{\frac{\Phi_{2}^{(12)}\left(\mathbf{k}^{\prime}, \mathbf{k}_{\mathbf{1}}\right)}{\mathbf{k}^{\prime 2}\left(\mathbf{k}_{\mathbf{1}}-\mathbf{k}^{\prime}\right)^{2}}\left[-\mathbf{k}_{\mathbf{1}}{ }^{2}+\frac{\mathbf{k}^{2}\left(\mathbf{k}_{\mathbf{1}}-\mathbf{k}^{\prime}\right)^{2}+\mathbf{k}^{\prime 2}\left(\mathbf{k}_{\mathbf{1}}-\mathbf{k}\right)^{2}}{\left(\mathbf{k}-\mathbf{k}^{\prime}\right)^{2}}\right]\right. \\
& \left.-\frac{\Phi_{2}^{(12)}\left(\mathbf{k}, \mathbf{k}_{\mathbf{1}}\right)}{\left(\mathbf{k}-\mathbf{k}^{\prime}\right)^{2}}\left[\frac{\mathbf{k}^{2}}{\mathbf{k}^{\prime 2}+\left(\mathbf{k}-\mathbf{k}^{\prime}\right)^{2}}+\frac{\left(\mathbf{k}_{\mathbf{1}}-\mathbf{k}\right)^{2}}{\left(\mathbf{k}_{\mathbf{1}}-\mathbf{k}^{\prime}\right)^{2}+\left(\mathbf{k}-\mathbf{k}^{\prime}\right)^{2}}\right]\right\}
\end{aligned}
$$

The details of impact factor calculations are given in the Appendices A-D. In the Appendix E we give some details used for performing the integration over the transversal component of the loop momenta.

\section{Discussion}

The quantities

$$
a_{\mathbf{O}}(\rho, \beta)=\frac{N}{\left(N^{2}-2\right)} \frac{I_{1}^{(3)}}{I_{0}^{(3)}}, \quad a_{\mathbf{I P}}(\rho, \beta)=N \frac{J_{1}^{(2)}}{J_{0}^{(2)}},
$$

may be interpreted as a partial contributions to the odderon and pomeron intercepts. Their dependence on $\rho, \beta$ is illustrated in Fig. 2 and Fig. 3. The jet's masses was supposed to be larger than $1 \mathrm{GeV}$.

The size of contributions to the polarized and unpolarized differential cross sections depends on the used jet model as well as on the choice of the vertices which describe the transition of the nucleon to the jet and on the choice of the vertex function which describes the conversion of one sort of jet to the jet of another sort. Because of the here used choice $V_{\mu}(k)=\gamma_{\mu}$, we are forced to put on the gauge conditions. Another possible choice, $V_{\mu}(k)=$ $\left[\gamma_{\mu}, \hat{k}\right] / M$, leads to the zero contribution to the asymmetry (in the limit of infinite large $s$ ).

We see that one-spin effect is rather large. Another mechanisms of onespin asymmetry associated with the nucleon resonances in intermediate state and final state interaction in $e p \rightarrow e p \pi$ process was considered in papers [4, 5] where the effect was of the same order.

\section{Acknowledgments}

The work was supported by INTAS-00366. E. Z. is gratefull to RFBR for the grant 0001-00617. 


\section{Appendix A}

The explicit expressions for the lowest order impact factors in the unpolarized case are

$$
\begin{aligned}
\Phi_{01}\left(\mathbf{k}_{\mathbf{1}}, \mathbf{p}\right) & =\frac{1}{4 s^{2}} S p\left(\hat{p}_{1}^{\prime}+m\right) O_{\mu}\left(\hat{p}_{1}+m\right) \tilde{O}_{\nu} p_{2}^{\mu} p_{2}^{\nu}, \\
\Phi_{02}\left(\mathbf{k}_{\mathbf{1}}\right) & =\frac{1}{4 s_{2}^{2}} S p\left(\hat{p}_{2}^{\prime}+M_{2}\right) \gamma_{\mu}\left(\hat{p}_{2}+m\right) \gamma_{\nu} k_{1 \perp}^{\mu} k_{1 \perp}^{\nu} .
\end{aligned}
$$

To simplify the calculation of the traces we write down here the useful relations

$$
\begin{gathered}
p_{1}^{\prime}=\frac{a_{1}}{s} q_{2}+\bar{\beta} q_{1}-p_{\perp}-k_{1 \perp}, \quad p_{1}^{\prime}+k_{1}=p_{1}-\frac{\mathbf{p}^{2}}{s} q_{2}-\beta q_{1}-p_{\perp}, \\
p_{1}-k_{1}=p_{1}+\frac{s_{1}}{s} q_{2}-k_{1 \perp}, \quad p_{2}^{\prime}=p_{2}+\frac{s_{2}}{s} q_{1}+k_{1 \perp}, \\
a_{1}=\left(M_{1}^{2}+\left(\mathbf{p}+\mathbf{k}_{\mathbf{1}}\right)^{2}\right) / \bar{\beta},
\end{gathered}
$$

which follow from the on mass shell conditions. Explicit expressions for $\Phi_{01}$ and $\Phi_{02}$ are

$$
\begin{aligned}
\Phi_{01}\left(\mathbf{k}_{\mathbf{1}}, \mathbf{p}\right) & =\frac{\beta^{4} \bar{\beta} \mathbf{k}_{\mathbf{1}}{ }^{2}}{2\left[\beta^{2} m^{2}+\mathbf{p}^{2}\right]\left[\beta^{2} m^{2}+\left(\mathbf{p}-\mathbf{k}_{\mathbf{1}} \beta\right)^{2}\right]} \\
\left.\Phi_{02} \mathbf{k}_{\mathbf{1}}\right) & =\frac{2 \mathbf{k}_{\mathbf{1}}{ }^{2}\left[\mathbf{k}_{\mathbf{1}}{ }^{2}+\left(M_{2}-m\right)^{2}\right]}{\left(\mathbf{q}^{2}+M_{2}^{2}-m^{2}\right)^{2}}
\end{aligned}
$$

\section{Appendix B}

The lowest order contribution to the impact factor corresponding to the polarized proton with 4 -momentum $p_{1}$ is

$$
\Phi_{11}=(1 / \bar{\beta}) \Phi_{1}^{b}+\Phi_{1}^{c}
$$

The quantity $\Phi_{1}^{b}$ has a form

$$
\begin{aligned}
\Phi_{1}^{b}= & \frac{1}{4 s^{3} R} S p\left(\hat{p}_{1}+m\right)\left(-\gamma_{5} \hat{a}\right) \tilde{O}_{\mu} p_{2}^{\mu}\left(\hat{p}_{1}^{\prime}+m\right) \hat{p}_{2} \\
& \times\left(\hat{p}_{1}^{\prime}+\hat{k}_{1}-\hat{k}+m\right) O_{1 \nu} p_{2}^{\nu} .
\end{aligned}
$$


The Sudakov decomposition of the 4 -vectors $p_{1}^{\prime}$ is given in (A.2). The exchanged gluon expansions are

$$
\begin{gathered}
k=\alpha q_{2}+k_{\perp}, \quad s \alpha=\mathbf{k}^{2}-d_{3}, \\
k_{1}=\alpha_{1} q_{2}+k_{1 \perp}, \quad s \alpha_{1}=\mathbf{k}_{\mathbf{1}}^{2}-d_{1},
\end{gathered}
$$

the quantity $O_{1 \mu}$ is equal

$$
O_{1 \mu}=\left(1 / d_{2}\right) \gamma_{\mu}\left(\hat{p}_{1}^{\prime}+\hat{k}_{1}+m\right) \gamma_{5}+\left(1 / d_{3}\right) \gamma_{5}\left(\hat{p}_{1}-\hat{p}_{1}+m\right) \gamma_{\mu}
$$

with $d_{1,2}$ given in $(11)$ and

$$
d_{3}=\frac{1}{\beta \bar{\beta}}\left[\beta^{2} m^{2}+(\mathbf{p}+\beta \mathbf{k})^{2}\right] .
$$

The quantity $\Phi_{1}^{c}$ has a form

$$
\begin{gathered}
\Phi_{1}^{c}=\frac{1}{4 s^{3} R} S p\left(\hat{p}_{1}+m\right)\left(-\gamma_{5} \hat{a}\right) \tilde{O}_{\mu} p_{2}^{\mu}\left(\hat{p}_{1}^{\prime}+m\right) O_{2 \nu} p_{2}^{\nu}\left(\hat{p}_{1}-\hat{k}+m\right) \hat{p}_{2}, \\
O_{2 \mu}=\gamma_{\mu} \frac{\hat{p}_{1}^{\prime}+\hat{k}_{1}-\hat{k}+m}{d_{4}} \gamma_{5}+\gamma_{5} \frac{\hat{p}_{1}-\hat{k}+m}{d_{1}} \gamma_{\mu},
\end{gathered}
$$

with

$$
d_{4}=-\frac{1}{\beta \bar{\beta}}\left[\beta^{2} m^{2}+(\mathbf{p}+\beta \mathbf{k})^{2}\right]
$$

and with the same expression for $p_{1}^{\prime}$.

The relevant representation for the exchanged gluon $4-$ momenta is following

$$
\begin{gathered}
k=\alpha q_{2}+k_{\perp}, \quad s \alpha=\mathbf{k}^{2} \\
k_{1}=\alpha_{1} q_{2}+k_{1 \perp}, \quad s \alpha_{1}=\mathbf{k}_{\mathbf{1}}{ }^{2}-d_{1}
\end{gathered}
$$

and the same expression for $p_{1}^{\prime}$.

Impact factor for the unpolarized proton $\Phi_{2}$ has a form

$$
\begin{aligned}
\Phi_{22}= & \frac{1}{s \beta s \beta_{1} s\left(\beta-\beta_{1}\right)} \frac{1}{4} S p\left(\hat{p}_{2}+m\right) \hat{k}_{1 \perp} \\
& \times\left(\hat{p}_{2}+\hat{k}_{1}+M_{2}\right)\left(\hat{k}_{1 \perp}-\hat{k}_{\perp}\right)\left(\hat{p}_{2}+\hat{k}+\tilde{M}_{2}\right) \hat{k}_{\perp} .
\end{aligned}
$$

For then calculation of the trace for $\Phi_{2}$ we used Sudakov representation

$$
\begin{gathered}
k=\beta q_{1}+k_{\perp}, \quad s \beta=\tilde{M}_{2}^{2}-m^{2}+\mathbf{k}^{2}, \\
k_{1}=\beta_{1} q_{1}+k_{1 \perp}, \quad s \beta_{1}=M_{2}^{2}-m^{2}+\mathbf{k}_{\mathbf{1}}{ }^{2} .
\end{gathered}
$$




\section{Appendix $\mathrm{C}$}

The impact factors for the case of unpolarized protons are

$$
\begin{gathered}
\Phi_{1}\left(\mathbf{k}_{\mathbf{1}}, \mathbf{p}\right)=\Phi_{01}\left(\mathbf{k}_{\mathbf{1}}, \mathbf{p}\right), \\
\Phi_{2}\left(\mathbf{k}^{\prime}\right)=\frac{1}{4 s s_{2}} S p\left(\hat{p}_{2}+\hat{k}_{\perp}^{\prime}+\beta^{\prime} q_{1}+M_{2}\right) \hat{p}_{1}\left(\hat{p}_{2}+m\right) \hat{k}_{\perp}^{\prime}
\end{gathered}
$$

where

$$
s_{2}=M_{2}^{2}-m^{2}+\mathbf{k}^{\prime 2} \text {. }
$$

\section{Appendix D}

Let us now give the expressions for the impact factors in the case of $\mathrm{RC}$ to the polarized cross sections. For the $\Phi_{1}^{(12)}$ in (21) we have

$$
\Phi_{1}^{(12)}\left(\mathbf{k}, \mathbf{k}_{\mathbf{1}}\right)=\Phi_{1 b}^{(12)}+\Phi_{1 c}^{(12)}
$$

with

$$
\begin{aligned}
\Phi_{1 b}^{(12)}= & \frac{1}{4 s R s_{11} s_{12}} S p\left(\hat{p}_{1}+m\right)\left(-\gamma_{5} \hat{a}\right) \tilde{O}_{\mu} p_{2}^{\mu}\left(\hat{p}_{1}^{\prime}+m\right) \\
& \times\left(\hat{k}_{1 \perp}-\hat{k}_{\perp}\right)\left(\hat{p}_{1}^{\prime}+\hat{k}_{1}-\hat{k}+m\right) O_{1 \nu} k_{\perp}^{\nu} ;
\end{aligned}
$$

and

$$
\begin{aligned}
\Phi_{1 c}^{(12)}= & \frac{1}{4 s R s_{21} s_{22}} S p\left(\hat{p}_{1}+m\right)\left(-\gamma_{5} \hat{a}\right) \tilde{O}_{\mu} p_{2}^{\mu}\left(\hat{p}_{1}^{\prime}+m\right) \\
& \times O_{2 \mu}\left(k_{1 \perp}-k_{\perp}\right)^{\mu}\left(\hat{p}_{1}-\hat{k}+m\right) \hat{k}_{\perp}
\end{aligned}
$$

with the substitutions similar to ones for $\Phi_{1}^{b}$ from Appendix B for the momenta including $\Phi_{1 b}^{(12)}$ and the substitutions similar to ones for $\Phi_{1}^{c}$ for $\Phi_{1 c}^{(12)}$. Besides

$$
\begin{gathered}
s_{11}=\left(\mathbf{k}_{\mathbf{1}}+\mathbf{p}\right)^{2}-(\mathbf{k}+\mathbf{p})^{2}, \quad s_{12}=m^{2}-\tilde{s}_{1}\left(m^{2}\right)-\mathbf{k}^{2} \\
s_{21}=-\mathbf{k}^{2}, \quad s_{22}=m^{2}-\tilde{s}_{1}+\mathbf{k}^{2}-\mathbf{k}_{\mathbf{1}}{ }^{2}
\end{gathered}
$$

The impact factor of unpolarized proton in this case have a form

$$
\begin{aligned}
\Phi_{2}^{(12)}\left(\mathbf{k}^{\prime}, \mathbf{k}_{\mathbf{1}}\right) & =\frac{1}{4 s_{2} s_{23} s_{13}} S p\left(\hat{p}_{2}+m\right) \hat{k}_{1 \perp}\left(\hat{p}_{2}+\hat{k}_{1 \perp}+\beta_{1} \hat{q}_{1}+M_{2}\right)(\mathrm{D} .5) \\
& \times\left(\hat{k}_{1 \perp}-\hat{k}_{\perp}^{\prime}\right)\left(\hat{p}_{2}+\hat{k}_{\perp}^{\prime}+\beta^{\prime} \hat{q}_{1}+\tilde{M}_{2}\right) \hat{k}_{\perp}^{\prime}
\end{aligned}
$$


with

$$
s_{13}=\tilde{M}_{2}^{2}-M_{2}^{2}-\mathbf{k}_{\mathbf{1}}{ }^{2}+\mathbf{k}^{\prime 2}, \quad s_{23}=\tilde{M}_{2}^{2}-m^{2}+\mathbf{k}^{\prime 2},
$$

and

$$
s \beta_{1}=M_{2}^{2}-m^{2}+\mathbf{k}_{\mathbf{1}}{ }^{2}, \quad s \beta^{\prime}=\tilde{M}_{2}^{2}-m^{2}+\mathbf{k}^{\prime 2} .
$$

\section{Appendix E}

Here we give some details used in performing the loop momenta integration.

When calculating the relevant trace we use the Shouthen identity

$\left(p_{1} p_{2} p_{3} p_{4}\right) Q_{\mu}=\left(\mu p_{2} p_{3} p_{4}\right) Q p_{1}+\left(p_{1} \mu p_{3} p_{4}\right) Q p_{2}+\left(p_{1} p_{2} \mu p_{4}\right) Q p_{3}+\left(p_{1} p_{2} p_{3} \mu\right) Q p_{4}$.

This identity permits to express all conversions with Levi-Chivita tensor in the standard form. For instance

$$
\left(p_{1} p_{2} k_{1} p_{\perp}\right)\left(a k_{2}\right)=\left(p_{1} p_{2} a p_{\perp}\right)\left(k_{1} k_{2}\right)+\left(p_{1} p_{2} k_{1} a\right)\left(p_{\perp} k_{2}\right) .
$$

The second term in the right side of this equation can be expressed through the

$$
E=\left(p_{1} p_{2} a p\right)=\frac{s}{2} m \rho R
$$

using the relation

$$
\int \frac{d^{2} \mathbf{k}}{\pi} \frac{d^{2} \mathbf{k}_{\mathbf{1}}}{\pi} \frac{d^{2} \mathbf{k}^{\prime}}{\pi} F\left(\mathbf{k}, \mathbf{k}_{\mathbf{1}}, \mathbf{k}^{\prime}, \mathbf{p}\right) \mathbf{k}_{\mathbf{i}}=\frac{\mathbf{p}_{\mathbf{i}}}{\mathbf{p}^{2}} \int \frac{d^{2} \mathbf{k}}{\pi} \frac{d^{2} \mathbf{k}_{\mathbf{1}}}{\pi} \frac{d^{2} \mathbf{k}^{\prime}}{\pi} F\left(\mathbf{k}, \mathbf{k}_{\mathbf{1}}, \mathbf{k}^{\prime}, \mathbf{p}\right) \mathbf{p} . \mathbf{k} .
$$

\section{References}

[1] H. Cheng and T. T. Wu, Phys. Rev. 182 (1969) 1852; G. Frolov and L. Lipatov, Yad. Fiz. 13 (1971), 588.

[2] I. Balitsky, L. N. Lipatov, Yad. Fiz. 28 (1978) 1596.

[3] V. S. Fadin, L. N. Lipatov and E. A. Kuraev, ZhETP 71 (1976) 840; ZhETP 72 (1977) 377. h/9802100.

[4] A. B. Arbuzov, V. A. Vasendina, E. A. Kuraev, V. A. Nikitin, Phys. Atom. Nucl. 57 (1994) 1004.

[5] A. Ahmedov, I. V. Akushevich, E. A. Kuraev, P. G. Ratcliffe, Eur. Phys. J. C11 (1999) 703. 